\title{
Commutative vs. Noncommutative Space Statistical Properties of Two-Dimensional Harmonic Oscillator in Magnetic Field
}

\author{
H. HASsAnABAdi ${ }^{a *}$, Z. DerAKhshani ${ }^{a}$ AND S. ZARRINKAMAR ${ }^{b}$ \\ ${ }^{a}$ Physics Department, Shahrood University of Technology, Shahrood, Iran \\ ${ }^{b}$ Department of Basic Sciences, Garmsar Branch, Islamic Azad University, Garmsar, Iran
}

(Received March 3, 2015; in final form June 8, 2015)

We consider the Schrödinger equation in presence of an external magnetic field in commutative and noncommutative spaces and by solving the equation in an exact analytical manner, report the statistical quantities of the system.

DOI: 10.12693/APhysPolA.129.3

PACS: 02.40.Gh, 03.65.-w, 03.65.Ge, 03.65.Pm

\section{Introduction}

The Schrödinger equation and its solutions are still a challenging subject in physics. Just as many other areas of science, where we have to solve a differential equation to obtain the required information, we have to first solve the building block of nonrelativistic quantum mechanics. In doing so, we have to use numerical and analytical techniques depending on the structure of the equation. In particular, the analytical approaches are attractive as they provide a deeper and more touchable insight into the physics of the problem [1-6]. Cooper et al. reviewed the theoretical formulation of supersymmetry quantum mechanics and discussed its applications in dealing with both relativistic and nonrelativistic equations of quantum mechanics [7]. Ciftci et al. used the asymptotic iteration method for finding solutions of the Schrödinger equation [8]. Slater considered a simplification of the Hartree-Fock method to analyze the related problems [9]. Stevenson applied the optimized perturbation theory to the field [10]. Dong et al. proposed the quasi-exact solutions of the Schrödinger equation via the ansatz technique which is a quasi-exact approach [11]. Another frequently used tool is the Nikiforov-Uvarov (NU) technique which transforms classes of equations of mathematical physics into hypergeometric form.

A reason of recent renewed interests in the wave equations of quantum mechanics is the implications of fundamental theories such as string theory. To be more precise, the noncommutative (NC) formulations of quantum mechanics and the proposition of the so-called minimal length, has motivated many recent studies on the quantum equations. The NC formulation, which is the focus of the present work, originates from fundamental theories

\footnotetext{
* corresponding author; e-mail: h.hasanabadi@shahroodut.ac.ir
}

such as string theory and quantum gravity which are still, and supposed to be for the coming years, open to debate. Seiberg and Witten extend earlier ideas about the appearance of $\mathrm{NC}$ geometry in string theory with a nonzero $B$-field [12]. Seiberg et al. studied strings in background electric fields with space-time non-commutativity [13]. Douglas and Nekrasov reviewed the generalization of field theory to NC space-time [14]. Connes et al. studied the toroidal compactification of matrix theory by using ideas coming from the results of NC geometry [15]. Pasquier introduced the ideas of NC geometry through the example of the quantum Hall effect [16].

Hassanabadi et al. considered the Klein-Gordon oscillator in the NC space (NCS) and NC phase-space with an extra pseudoharmonic potential in presence and absence magnetic field [17]. Santos et al. obtained the thermodynamical properties of graphene in NC phase-space [18]. Until now, various concepts of quantum mechanics have been studied within this framework. This list includes quantum Hall effect, graphene system, etc. [16, 18]. Also, Scholtz et al. studied the formulation, interpretation and application of non-commutative quantum mechanics [19]. Scholtz et al. obtained the spectrum of the NC spherical well and discussed the infinite and finite cases in two dimensions [20]. Mandal and Rai showed the behavior of the $(2+1)$-dimensional NC Dirac oscillator in an external magnetic field [21]. Scholtz and Govaerts calculated the thermodynamic properties of a $\mathrm{NC}$ fermion gas [22]. Gamboa et al. worked on the NC quantum mechanics in two-dimensional central field and found a smooth limit for small values of $\theta$ and for non-polynomial ones [23]. In our work, we are going to consider the NC Schrödinger equation with a harmonic potential which is of both research and pedagogical interests. Having calculated the solutions of the problem in an exact analytical manner, we next intend to calculate the statistical properties of the system including the partition function, internal energy, chemical potential and entropy. 


\section{The noncommutative space}

We first review the basic formulae of NC algebra. In the commutative quantum mechanics, the position and momentum operators satisfy the Heisenberg algebra [24]:

$$
\left[x_{i}, x_{j}\right]=\left[p_{i}, p_{j}\right]=0,\left[x_{i}, p_{j}\right]=\mathrm{i} \delta^{i j},
$$

At very tiny scales such as the string scale, the commutation relations ought to be modified and in the so-called NCS, the commutation relations appear in the form

$$
\left[x_{i}, x_{j}\right]=\mathrm{i} \theta^{i j}, \quad\left[p_{i}, p_{j}\right]=0, \quad\left[x_{i}, p_{j}\right]=\mathrm{i} \delta^{i j},
$$

where $\theta \varepsilon^{i j}$, with $\varepsilon^{i j}$ being an anti-symmetric tensor and the NC parameter $\theta$ is assumed to be extremely small. To map the ordinary quantum mechanics to its NC version, we apply the shifts

$$
\begin{aligned}
& x \rightarrow x-\frac{\theta}{2} p_{y}, \quad y \rightarrow p+\frac{\theta}{2} p_{x}, \\
& p_{x} \rightarrow p_{x}+\frac{\theta}{2} y, \quad p_{y} \rightarrow p_{y}-\frac{\theta}{2} x,
\end{aligned}
$$

which are called the Bopp-shift in the jargon. In the $\mathrm{NC}$ formulation, for arbitrary $f$ and $g$ functions, we have to define the Moyal product

$$
(f * g)(x)=\exp \left(\mathrm{i} \theta^{i j} \partial x^{i} \partial x^{j}\right) f\left(x^{i}\right) g\left(x^{j}\right)
$$

In this case, the Moyal-Weyl product can be replaced by a Bopp shift [25].

\section{Schrödinger equation in the presence of magnetic field in commutative space}

Let us first recall that to analyze a charged particle in a magnetic field, the momentum is transformed as $\boldsymbol{p} \rightarrow\left(\boldsymbol{p}-\frac{e}{c} \boldsymbol{A}\right)$. The Hamiltonian in the presence of magnetic field in commutative space with the oscillatory interaction term is

$$
\begin{aligned}
& H^{(c)}=\frac{1}{2 M}\left[\left(p_{x}-\frac{e}{c} A_{x}\right) \hat{i}+\left(p_{y}-\frac{e}{c} A_{y}\right) \hat{j}\right]^{2} \\
& \quad+\frac{M}{2} \omega^{2}\left(x^{2}+y^{2}\right)=\frac{1}{2 M}\left[p_{x}^{2}+\frac{\mathrm{e}^{2}}{c^{2}} A_{x}^{2}-\frac{e}{c} p_{x} A_{x}\right. \\
& \quad-\frac{e}{c} A_{x} p_{x}+p_{y}^{2}+\frac{\mathrm{e}^{2}}{c^{2}} A_{y}^{2} \\
& \left.\quad-\frac{e}{c} p_{y} A_{y}-\frac{e}{c} A_{y} p_{y}\right]+\frac{M}{2} \omega^{2}\left(x^{2}+y^{2}\right), \\
& A=\left(-\frac{1}{2} B y, \frac{1}{2} B x, 0\right) .
\end{aligned}
$$

Bearing in mind $L_{z}=x p_{y}-y p_{x}$, Eq. (5) appears as

$$
\begin{aligned}
& {\left[\left(p_{x}^{2}+p_{y}^{2}\right)+\left(\frac{e^{2} B^{2}}{4 c^{2}}+M^{2} \omega^{2}\right)\left(x^{2}+y^{2}\right)-\frac{e B}{c} L_{z}\right] \psi=} \\
& \quad 2 M E_{n, m}^{(c)} \psi,
\end{aligned}
$$

or

$$
\begin{aligned}
& {\left[\frac{\mathrm{d}^{2}}{\mathrm{~d} r^{2}}+\frac{1}{r} \frac{\mathrm{d}}{\mathrm{d} r}-\frac{m^{2}}{r^{2}}+\left(\frac{e^{2} B^{2}}{4 c^{2}}+M^{2} \omega^{2}\right) r^{2}-\frac{e B}{c} L_{z}\right] \psi=} \\
& 2 M E_{n, m}^{(c)} \psi .
\end{aligned}
$$

Using the transformation $r^{2}=s$, Eq. (8) comes into the form

$$
\left\{\frac{\mathrm{d}^{2}}{\mathrm{~d} s^{2}}+\frac{1}{s} \frac{\mathrm{d}}{\mathrm{d} s}+\frac{1}{s^{2}}\left[-\frac{m^{2}}{4}+\left(\frac{e^{2} B^{2}}{16 c^{2}}+\frac{M^{2}}{4} \omega^{2}\right) s^{2}\right.\right.
$$

$$
\left.\left.-\left(\frac{e B}{4 c} L_{z}+\frac{M E_{n, m}^{(c)}}{2}\right) s\right]\right\} \psi=0
$$

The latter can be simply solved by the powerful NU method with the required parameters being [26]:

$$
\begin{aligned}
& \alpha_{1}=1, \quad \alpha_{2}=\alpha_{3}=0, \quad \alpha_{4}=\alpha_{5}=0, \\
& \xi_{1}=-\frac{e^{2} B^{2}}{16 c^{2}}-\frac{M^{2}}{4} \omega^{2}, \quad \xi_{2}=-\frac{e B}{4 c} L_{z}-\frac{M E_{n, m}^{(c)}}{2}, \\
& \xi_{3}=\frac{1}{4} m^{2}, \quad \alpha_{8}=\xi_{3}=\frac{1}{4} m^{2},
\end{aligned}
$$

and

$$
\begin{aligned}
& \alpha_{6}=-\frac{e^{2} B^{2}}{16 c^{2}}-\frac{M^{2}}{4} \omega^{2}, \quad \alpha_{7}=\frac{e B}{4 c} L_{z}+\frac{M E_{n, m}^{(c)}}{2}, \\
& \alpha_{9}=-\frac{e^{2} B^{2}}{16 c^{2}}-\frac{M^{2}}{4} \omega^{2} .
\end{aligned}
$$

Using the NU technique, and substituting $\sqrt{-\alpha_{9}}$ as $Q$, the energy relation for $L_{z}=m \hbar$ and $\hbar=1$ is

$$
-(2 n+1) Q+\frac{e B}{4 c} L_{z}+\frac{M E_{n, m}^{(c)}}{2}-m Q=0,
$$

which gives the explicit form of the energy as

$$
E_{n, m}^{(c)}=(2 n+1) \frac{2}{M} Q+\frac{4 m}{M} Q-\frac{2 e B}{4 M c} m .
$$

The latter, in the case of $m=0$, yields

$$
E_{n}^{(c)}=\left(n+\frac{1}{2}\right) \frac{4 Q}{M} .
$$

Recalling the energy relation of the ordinary nonrelativistic harmonic oscillator, we find the corresponding frequency as

$$
\omega^{(c)}=\frac{4}{M} \sqrt{\frac{e^{2} B^{2}}{16 c^{2}}+\frac{M^{2}}{4} \omega^{2}} .
$$

\section{Statistical properties in commutative space}

The partition function of the system in the CS is

$$
Z_{1}^{(c)}=\sum_{n=0}^{\infty} \mathrm{e}^{-\beta\left(n+\frac{1}{2}\right) \omega^{(c)}},
$$

which gives

$$
Z^{(c)}=\left(Z_{1}^{(c)}\right)^{N}=\left[2 \sinh \left(\frac{4 \beta}{M} Q\right)\right]^{-N} .
$$

where $\beta=\frac{1}{k_{\mathrm{B}} T}$ and $k_{\mathrm{B}}$ is the Boltzmann constant. Having calculated the partition function, we now calculate other statistical properties of the system. The internal energy of the system is

$$
\begin{aligned}
& U^{(c)}=-\frac{\partial}{\partial \beta} \ln Z^{(c)}=-\frac{\partial}{\partial \beta} \ln \left(\mathrm{e}^{\frac{\beta \omega}{2}(c)}-\mathrm{e}^{-\frac{\beta \omega}{2}(c)}\right)^{-N}= \\
& \frac{2 N}{M} Q \operatorname{coth}\left(\frac{2 \beta}{M} Q\right) .
\end{aligned}
$$

In the extreme limit where $T \rightarrow \infty$, from $\operatorname{coth}(y) \cong$ $\frac{1}{y}=\frac{2}{\beta \omega^{(c)}}$ and Eq. (17) reduces to the well-known relation

$$
U^{(c)}=N k_{\mathrm{B}} T \text {. }
$$

The Helmholtz free energy is found to be

$$
A^{(c)}=-k_{\mathrm{B}} T \ln Z^{(c)}=N k_{\mathrm{B}} T \ln \left(2 \sinh \left(\frac{2 \beta}{M} Q\right)\right)
$$

The chemical potential of the system is 


$$
\mu^{(c)}=\left(\frac{\partial A^{(c)}}{\partial N}\right)_{V, T}=k_{\mathrm{B}} T \ln \left(2 \sinh \left(\frac{2 \beta}{M} Q\right)\right) .
$$

and the entropy takes the form

$$
\begin{aligned}
& S^{(c)}=-\left(\frac{\partial A^{(c)}}{\partial T}\right)_{V, N}=-N k_{\mathrm{B}}\left[\ln \left(2 \sinh \left(\frac{2 \beta}{M} Q\right)\right)\right. \\
& \left.\quad-\left(\frac{2 \beta}{M} Q\right) \operatorname{coth}\left(\frac{2 \beta}{M} Q\right)\right] .
\end{aligned}
$$

\section{Schrödinger equation in the presence of magnetic field in noncommutative space}

To consider the problem in the NCS, we consider the transformations

$$
\begin{aligned}
& x \rightarrow x-\frac{\theta}{2} p_{y}, \quad y \rightarrow p+\frac{\theta}{2} p_{x}, \quad p_{x} \rightarrow p_{x}+\frac{\theta}{2} y, \\
& p_{y} \rightarrow p_{y}-\frac{\theta}{2} x
\end{aligned}
$$

and the commutation relation between the space and momentum is

$$
\left[x_{i}, p_{j}\right]=\mathrm{i} \delta^{i j}
$$

Summing up the above relations, we have

$$
\begin{aligned}
& H^{(N C)}=\frac{1}{2 M}\left[\left(p_{x}-\frac{e}{c} A_{x}\right) \hat{i}+\left(p_{y}-\frac{e}{c} A_{y}\right) \hat{j}\right]^{2} \\
& \quad+\frac{M}{2} \omega^{2}\left(x^{2}+y^{2}\right)=\frac{1}{2 M}\left[p_{x}^{2}+\frac{\theta^{2}}{4} y^{2}+\theta p_{x} y\right. \\
& \quad+\frac{e^{2} B^{2}}{4 c^{2}}\left(y^{2}+\frac{\theta^{2}}{4} p_{x}^{2}+\theta y p_{x}\right)+\frac{e B}{2 c}\left(y+\frac{\theta}{2} p_{x}\right) \\
& \quad \times\left(p_{x}+\frac{\theta}{2} y\right)+\frac{e B}{2 c}\left(p_{x}+\frac{\theta}{2} y\right)\left(y+\frac{\theta}{2} p_{x}\right) \\
& \quad+p_{y}^{2}+\frac{\theta^{2}}{4} x^{2}-\theta p_{y} x+\frac{e^{2} B^{2}}{4 c^{2}}\left(x^{2}+\frac{\theta^{2}}{4} p_{y}^{2}-\theta x p_{y}\right) \\
& \quad-\frac{e B}{2 c}\left(p_{y}-\frac{\theta}{2} x\right)\left(x-\frac{\theta}{2} p_{y}\right) \\
& \left.\quad-\frac{e B}{2 c}\left(x-\frac{\theta}{2} p_{y}\right)\left(p_{y}-\frac{\theta}{2} x\right)\right] \\
& \quad+\frac{M \omega^{2}}{2}\left(x^{2}+y^{2}+\frac{\theta^{2}}{4}\left(p_{x}^{2}+p_{y}^{2}\right)+\theta\left(y p_{x}-x p_{y}\right)\right),
\end{aligned}
$$

or

$$
\begin{aligned}
& {\left[\left(1+\frac{e^{2} B^{2} \theta^{2}}{16 c^{2}}+\frac{e B \theta}{2 c}+\frac{M^{2}}{4} \omega^{2} \theta^{2}\right) p^{2}\right.} \\
& +\left(\frac{\theta^{2}}{4}+\frac{e^{2} B^{2}}{4 c^{2}}+\frac{e B \theta}{2 c}+M^{2} \omega^{2}\right) r^{2} \\
& -\left(2 \theta+\frac{e^{2} B^{2} \theta}{4 c^{2}}+\frac{e B}{c}+\frac{e B \theta^{2}}{4 c}\right) L_{z} \\
& \left.-2 M E_{n, m}^{(N C)}\right] \psi=0 .
\end{aligned}
$$

Using

$$
\begin{aligned}
& \alpha=1+\frac{e^{2} B^{2} \theta^{2}}{16 c^{2}}+\frac{e B \theta}{2 c}+\frac{M^{2}}{4} \omega^{2} \theta^{2}, \\
& \eta=\frac{\theta^{2}}{4}+\frac{e^{2} B^{2}}{4 c^{2}}+\frac{e B \theta}{2 c}+M^{2} \omega^{2}, \\
& \gamma=2 \theta+\frac{e^{2} B^{2} \theta}{4 c^{2}}+\frac{e B}{c}+\frac{e B \theta^{2}}{4 c},
\end{aligned}
$$

Eq. (25) takes the form

$$
\begin{aligned}
\alpha p^{2}+\nu r^{2}-\gamma L_{z}-2 M E_{n, m}^{(N C)} & =0, \\
\text { or } \quad & p^{2}+\frac{\nu}{\alpha} r^{2}-\frac{\gamma}{\alpha} L_{z}-\frac{2}{\alpha} M E_{n, m}^{(N C)}=0,
\end{aligned}
$$

which corresponds to the differential equation

$$
\begin{aligned}
& \frac{\mathrm{d}^{2}}{\mathrm{~d} s^{2}}+\frac{\mathrm{d}}{s \mathrm{~d} s} \\
& \quad+\left[-\frac{m^{2}}{4}+\frac{\eta s^{2}}{4 \alpha}-\left(\frac{\gamma}{4 \alpha} L_{z}-\frac{2 M E_{n, m}^{(N C)}}{4 \alpha}\right) s\right] / s^{2}=0,
\end{aligned}
$$

possessing the $\mathrm{NU}$ parameters

$$
\begin{aligned}
& \alpha_{1}=1, \quad \alpha_{2}=\alpha_{3}=0, \quad \alpha_{4}=0, \quad \alpha_{5}=0, \\
& \xi_{1}=-\frac{\eta}{4 \alpha}, \quad \xi_{2}=-\frac{\gamma}{4 \alpha} L_{z}-\frac{M E_{n, m}^{(N C)}}{2 \alpha}, \quad \xi_{3}=\frac{m^{2}}{4}, \\
& \alpha_{6}=\xi_{1}=-\frac{\eta}{4 \alpha} \alpha_{7}=-\xi_{2}=\frac{\gamma}{4 \alpha} L_{z}+\frac{M E_{n, m}^{(N C)}}{2 \alpha}, \\
& \alpha_{8}=\xi_{3}=\frac{m^{2}}{4}, \quad \alpha_{9}=\alpha_{6}=-\frac{\eta}{4 \alpha} .
\end{aligned}
$$

Therefore, the energy is

$$
\begin{aligned}
& E_{n, m}^{(N C)}=\frac{2 \alpha}{M}(2 n+1) \sqrt{\frac{\eta}{4 \alpha}}+\frac{2 \alpha m}{M} \sqrt{\frac{\eta}{4 \alpha}} \\
& -\frac{2 \alpha}{M} \frac{\gamma}{4 \alpha} L_{z},
\end{aligned}
$$

which, for $L_{z}=m \hbar$ and $\hbar=1$, takes the form

$$
\begin{aligned}
& E_{n, m}^{(N C)}=\frac{2 \alpha}{M}(2 n+1) \sqrt{\frac{\eta}{4 \alpha}}+\frac{\alpha m}{M} \sqrt{\frac{\eta}{\alpha}} \\
& \quad-\frac{\gamma m}{2 M} .
\end{aligned}
$$

The latter, in the case of $m=0$, gives

$$
\begin{aligned}
& E_{n}^{(N C)}=\left(n+\frac{1}{2}\right) \\
& \quad \times \frac{4 R}{M}\left(1+\frac{e^{2} B^{2} \theta^{2}}{16 c^{2}}+\frac{e B \theta}{2 c}+\frac{M^{2}}{4} \omega^{2} \theta^{2}\right),
\end{aligned}
$$

where $R=\sqrt{\frac{\theta^{2} c^{2}+e^{2} B^{2}+4 M^{2} \omega^{2} c^{2}+2 e B \theta c}{16 c^{2}+e^{2} B^{2} \theta^{2}+8 e B \theta c+4 M^{2} \omega^{2} \theta^{2} c^{2}}}$. A comparison with the energy relation of the harmonic oscillator, gives the corresponding frequency as

$$
\omega^{(N C)}=\frac{4 R}{M}\left(1+\frac{e^{2} B^{2} \theta^{2}}{16 c^{2}}+\frac{e B \theta}{2 c}+\frac{M^{2}}{4} \omega^{2} \theta^{2}\right),
$$

Here, if we put $\theta=0, \omega^{(N C)}$ exactly gives $\omega^{(C)}$.

\section{Statistical properties in noncommutative space}

Now, for calculation of the partition function in NCS, we have

$$
\begin{gathered}
Z_{1}^{(N C)}=\sum_{n=0}^{\infty} \mathrm{e}^{-\beta\left(n+\frac{1}{2}\right) \omega^{(N C)}}=\left[2 \sinh \left(\frac{\beta}{2} \omega^{(N C)}\right)\right]^{-1}= \\
{\left[2 \sinh \left(\frac{2 \beta R}{M}\left(1+\frac{e^{2} B^{2} \theta^{2}}{16 c^{2}}+\frac{e B \theta}{2 c}+\frac{M^{2} \omega^{2} \theta^{2}}{4}\right)\right)\right]^{-1},}
\end{gathered}
$$

which gives

$$
\begin{aligned}
& Z^{(N C)}=\left(Z_{1}^{(N C)}\right)^{N}= \\
& \frac{1}{2 \sinh \left(\frac{2 \beta R}{M}\left(1+\frac{e^{2} B^{2} \theta^{2}}{16 c^{2}}+\frac{e B \theta}{2 c}+\frac{M^{2} \omega^{2} \theta^{2}}{4}\right)\right)^{N}} .
\end{aligned}
$$


The latter, for $\theta=0$, gives Eq. (16). Let us now calculate some useful statistical quantities. The internal energy for this system is

$$
\begin{gathered}
U^{(N C)}=-\frac{\partial}{\partial \beta} \ln Z^{(N C)}= \\
-\frac{\partial}{\partial \beta} \ln \left(\mathrm{e}^{\frac{\beta}{2} \omega^{(N C)}}-\mathrm{e}^{-\frac{\beta}{2} \omega^{(N C)}}\right)^{-N}= \\
\frac{N \omega^{(N C)}}{2} \operatorname{coth}\left(\frac{\beta \omega^{(N C)}}{2}\right)= \\
\frac{2 N R}{M}\left(1+\frac{e^{2} B^{2} \theta^{2}}{16 c^{2}}+\frac{e B \theta}{2 c}+\frac{M^{2}}{4} \omega^{2} \theta^{2}\right) \\
\times \operatorname{coth}\left(\frac{2 \beta}{M}\left(1+\frac{e^{2} B^{2} \theta^{2}}{16 c^{2}}+\frac{e B \theta}{2 c}+\frac{M^{2}}{4} \omega^{2} \theta^{2}\right)\right) .
\end{gathered}
$$

The Helmholtz free energy in the NCS in this case appears as

$$
A^{(N C)}=-k_{\mathrm{B}} T \ln Z^{(N C)}=
$$

$N k_{\mathrm{B}} T \ln \left(2 \sinh \left(\frac{2 \beta R}{M}\left(1+\frac{e^{2} B^{2} \theta^{2}}{16 c^{2}}+\frac{e B \theta}{2 c}+\frac{M^{2}}{4} \omega^{2} \theta^{2}\right)\right)\right)$, and the chemical potential takes the form

$$
\mu^{(N C)}=\left(\frac{\partial A^{(N C)}}{\partial N}\right)_{V, T}=
$$

$k_{\mathrm{B}} T \ln \left(2 \sinh \left(\frac{2 \beta R}{M}\left(1+\frac{e^{2} B^{2} \theta^{2}}{16 c^{2}}+\frac{e B \theta}{2 c}+\frac{M^{2}}{4} \omega^{2} \theta^{2}\right)\right)\right)$.

As the last part, we report the entropy of the NC case as

$$
\begin{gathered}
S^{(N C)}=-\left(\frac{\partial A^{(N C)}}{\partial T}\right)_{V, N}= \\
-N k_{\mathrm{B}}\left[\operatorname { l n } \left(2 \sinh \left(\frac{2 \beta R}{M}\left(1+\frac{e^{2} B^{2} \theta^{2}}{16 c^{2}}+\frac{e B \theta}{2 c}+\frac{M^{2}}{4} \omega^{2} \theta^{2}\right)\right)\right.\right. \\
-\frac{2 \beta R}{M}\left(1+\frac{e^{2} B^{2} \theta^{2}}{16 c^{2}}+\frac{e B \theta}{2 c}+\frac{M^{2}}{4} \omega^{2} \theta^{2}\right) \\
\left.\left.\operatorname{coth}\left(\frac{2 \beta R}{M}\left(1+\frac{e^{2} B^{2} \theta^{2}}{16 c^{2}}+\frac{e B \theta}{2 c}+\frac{M^{2}}{4} \omega^{2} \theta^{2}\right)\right)\right)\right] .
\end{gathered}
$$

Here, by inserting $\theta=0$, Eq. (40) yields Eq. (21).

\section{Conclusions}

We solved the Schrödinger equation in the presence of magnetic field in commutative and non-commutative spaces. By obtaining the exact analytical wave function and the energy relation in both spaces via the NU method, we calculated the partition function and thereby the statistical quantities including the internal energy, the Helmholtz free energy, chemical potential and the entropy. Having found the energy, the splitting and the shifts due to the NC parameter is explicitly seen. In addition, we see that while the energy difference for successive quantum number $m$ in the commutative case is

$$
E_{n, m+1}^{(c)}-E_{n, m}^{(c)}=\frac{2}{M} \sqrt{\left(\frac{e^{2} B^{2}}{16 c^{2}}+\frac{M^{2}}{4} \omega^{2}\right)}-\frac{2 e B}{4 M c}
$$

The corresponding difference in the noncommutative case is

$$
E_{n, m+1}^{(N C)}-E_{n, m}^{(N C)}=E_{n, m+1}^{(C)}-E_{n, m}^{(C)}
$$

$$
\begin{aligned}
& +\frac{Q}{M}\left(\frac{\theta^{2} c^{2}+2 e B \theta c}{e^{2} B^{2}+4 M^{2} \omega^{2} c^{2}}+\frac{e^{2} B^{2} \theta^{2}}{16 c^{2}}+\frac{e B \theta}{2 c}+\frac{M^{2}}{4} \omega^{2} \theta^{2}\right. \\
& \left.+\left[\frac{e^{2} B^{2} \theta^{2}}{16 c^{2}}+\frac{e B \theta}{2 c}+\frac{M^{2}}{4} \omega^{2} \theta^{2}\right] \frac{\theta^{2} c^{2}+2 e B \theta c}{e^{2} B^{2}+4 M^{2} \omega^{2} c^{2}}\right) \\
& -\frac{1}{2 M}\left(2 \theta+\frac{e^{2} B^{2} \theta}{4 c^{2}}+\frac{e B \theta^{2}}{4 c}\right) .
\end{aligned}
$$

That explicitly shows the shift

$$
\begin{aligned}
& E_{n, m}^{(N C)}=E_{n, m}^{(C)}+\frac{(2 n+1)+m}{M} Q\left(\frac{\theta^{2} c^{2}+2 e B \theta c}{e^{2} B^{2}+4 M^{2} \omega^{2} c^{2}}\right. \\
& +\frac{e^{2} B^{2} \theta^{2}}{16 c^{2}}+\frac{e B \theta}{2 c}+\frac{M^{2}}{4} \omega^{2} \theta^{2} \\
& \left.+\left[\frac{e^{2} B^{2} \theta^{2}}{16 c^{2}}+\frac{e B \theta}{2 c}+\frac{M^{2}}{4} \omega^{2} \theta^{2}\right] \frac{\theta^{2} c^{2}+2 e B \theta c}{e^{2} B^{2}+4 M^{2} \omega^{2} c^{2}}\right) \\
& \quad-\frac{m}{2 M}\left(2 \theta+\frac{e^{2} B^{2} \theta}{4 c^{2}}+\frac{e B \theta^{2}}{4 c}\right)
\end{aligned}
$$

due to noncommutative formulation.

\section{Acknowledgments}

It is a great pleasure for authors to thank the referee for useful comments on the manuscript.

\section{Appendix: The Nikiforov-Uvarov method}

The parametric form of the Nikiforov-Uvarov method takes the form [27]:

$$
\begin{aligned}
& \frac{\mathrm{d}^{2} \psi(s)}{\mathrm{d} s^{2}}+\frac{\alpha_{1}-\alpha_{2} s}{s\left(1-\alpha_{3} s\right)} \frac{d \psi(s)}{\mathrm{d} s}+\frac{1}{s^{2}\left(1-\alpha_{3} s\right)^{2}} \\
& \quad \times\left\{-\xi_{1} s^{2}+\xi_{2} s-\xi_{3}\right\} \psi(s)=0 .
\end{aligned}
$$

The energy eigenvalues equation and eigenfunctions respectively satisfy the following sets of equation:

$$
\begin{aligned}
& \alpha_{2} n-(2 n+1) \alpha_{5}+(2 n+1)\left(\sqrt{\alpha_{9}}+\alpha_{3} \sqrt{\alpha_{8}}\right) \\
& \quad+n(n-1) \alpha_{3}+\alpha_{7}+2 \alpha_{3} \alpha_{8}+2 \sqrt{\alpha_{8} \alpha_{9}}=0, \\
& \psi(s)=s^{\alpha_{12}}\left(1-\alpha_{3} s\right)^{-\alpha_{12}-\left(\alpha_{13} / \alpha_{3}\right)} \\
& \quad \times P_{n}^{\left(\alpha_{10}-1,\left(\alpha_{11} / \alpha_{3}\right)-\alpha_{10}-1\right)}\left(1-2 \alpha_{3} s\right),
\end{aligned}
$$

where

$$
\begin{aligned}
& \alpha_{4}=\frac{1}{2}\left(1-\alpha_{1}\right), \quad \alpha_{5}=\frac{1}{2}\left(\alpha_{2}-2 \alpha_{3}\right), \\
& \alpha_{6}=\alpha_{5}^{2}+\xi_{1}, \quad \alpha_{7}=2 \alpha_{4} \alpha_{5}-\xi_{2}, \\
& \alpha_{8}=\alpha_{4}^{2}+\xi_{3}, \quad \alpha_{9}=\alpha_{3} \alpha_{7}+\alpha_{3}^{2} \alpha_{8}+\alpha_{6}, \\
& \alpha_{10}=\alpha_{1}+2 \alpha_{4}+2 \sqrt{\alpha_{8}}, \\
& \alpha_{11}=\alpha_{2}-2 \alpha_{5}+2\left(\sqrt{\alpha_{9}}+\alpha_{3} \sqrt{\alpha_{8}}\right), \\
& \alpha_{12}=\alpha_{4}+\sqrt{\alpha_{8}}, \quad \alpha_{13}=\alpha_{5}-\left(\sqrt{\alpha_{9}}+\alpha_{3} \sqrt{\alpha_{8}}\right)(\mathrm{A} .4)
\end{aligned}
$$
and $P_{n}$ is the orthogonal Jacobi polynomial.

\section{References}

[1] G.F. Wei, S.H. Dong, Can. J. Phys. 89, 1225 (2011).

[2] G.F. Wei, S.H. Dong, Eur. Phys. J. A 46, 207 (2010).

[3] S.H. Dong, J. Garcia-Ravelo, J. Phys. Scr. 75, 307 (2007) 
[4] S.H. Dong, Int. J. Theor. Phys. 39, 1119 (2000).

[5] S.H. Dong, Int. J. Theor. Phys. 40, 559 (2001).

[6] A.F. Nikiforov, V.B. Uvarov, Special Functions of Mathematical Physics, Birkhäuser, Basel 1988.

[7] F. Cooper, A. Khare, U. Sukhatme, Phys. Rep. 251, 267 (1995).

[8] H. Ciftci, R.L. Hall, N. Saad, J. Phys. A Math. Theor. 36, 11807 (2003).

[9] J.C. Slater, Phys. Rev. 81, 385 (1951).

[10] P.M. Stevenson, Phys. Rev. D 23, 2916 (1981).

[11] S.H. Dong, Z. Ma, G. Espozito, Found. Phys. Lett. 12, 465 (1999).

[12] N. Seiberg, E. Witten, J. High Energy Phys. 09, 032 (1999).

[13] N. Seiberg, L. Susskind, N. Toumbas, J. High Energy Phys. 06, 044 (2000).

[14] M.R. Douglas, N.A. Nekrasov, Rev. Mod. Phys. 73, 977 (2001).

[15] A. Connes, M.R. Douglas, A. Schwarz, J. High Energy Phys. 02, 003 (1998).

[16] V. Pasquier, Seminaire Poincaré X, 1 (2007).
[17] H. Hassanabadi, S.S. Hosseini, Z. Molaee, Commun. Theor. Phys. 60, 9 (2013).

[18] V. Santos, R.V. Malufy, C.A.S. Almeida, arXiv:1401.8051v1.

[19] F.G. Scholtz, L. Gouba, A. Hafver, C.M. Rohwer, J. Phys. A Math. Theor. 42, 175303 (2009).

[20] F.G. Scholtz, B. Chakraborty, J. Govaerts, S. Vaidya, arXiv:0709.3357v1 [hep-th] 21 Sep 20.

[21] B.P. Mandal, S.K. Rai, Phys. Lett. A 376, 2467 (2012).

[22] F.G. Scholtz, J. Govaerts, arXiv:0810.3064v1 [hep-th] 17 Oct 2008.

[23] J. Gamboa, M. Loewe, F. Méndez, J.C. Rojas, Int. J. Mod. Phys. A 17, 2555 (2002).

[24] H. Hassanabadi, S.S. Hosseini, A. Boumali, S. Zarrinkamar, J. Math. Phys. 55, 033502 (2014).

[25] T. Curtright, D. Fairlie, C. Zachos, Phys. Rev. D 58, 025002 (1998).

[26] E. Maghsoodi, H. Hassanabadi, S. Zarrinkamar, Chin. Phys. B 22, 030302 (2013).

[27] C. Tezcan, R. Sever, Int. J. Theor. Phys. 48, 337 (2009). 